\title{
Stratospheric Sudden Warming with Projected Global Warming and Related Tropospheric Wave Activity
}

\author{
Masaru Inatsu', Masahide Kimoto ${ }^{2}$, and Akimasa Sumi ${ }^{3}$ \\ ${ }^{1}$ Graduate School of Science, Hokkaido University, Sapporo, Japan \\ ${ }^{2}$ Center for Climate System Research, The University of Tokyo, Tokyo, Japan \\ ${ }^{3}$ Integrated Research System for Sustainability Science, The University of Tokyo, Tokyo, Japan
}

\begin{abstract}
Stratospheric sudden warming (SSW) in association with projected global warming $(\mathrm{GW})$ in the Northern Hemisphere was investigated by 20th-century and 21 st-century simulations (20CS and $21 \mathrm{CS}$ ) using the atmosphere-ocean coupled general circulation model. Composite analysis revealed that the model simulated 20th-century dynamical aspects of SSW well. Although SSW events in early winter are missing, considering difficulties in reasonable SSW simulation, we regard the $20 \mathrm{CS}$ results as reference values. The $21 \mathrm{CS}$ suggests that forcing by GW increases the number of both major and minor SSW events in Dec, Jan, and early Feb, in correlation with larger eddy heat flux $(\mathrm{EHF})$ at $100 \mathrm{hPa}$ in Nov, Dec, and early Jan. The increase of the flux mainly originates from tropospheric stationary wave response. The greater flux continues until early Jan and then the polar night jet is much more weakened in Feb. This background state then effectively blocks wave propagation into the stratosphere and the EHF at $100 \mathrm{hPa}$ slightly decreases though the difference is not statistically significant. The major SSW events decreases in Mar.
\end{abstract}

\section{Introduction}

An abrupt stratospheric temperature jump, known as stratospheric sudden warming (SSW), was first observed in the early 1950s and has been explained by the theory of wave-mean flow interaction (Matsuno 1971). Large-amplitude Rossby waves propagating from the troposphere tend to trigger SSW.

The effects of projected global warming (GW) on SSW have been discussed since the 1990s. Rind et al. (1990) suggested that double-carbon dioxide $\left(\mathrm{CO}_{2}\right)$ forcing tends to result in the earlier timing of SSW. However, although Rind et al. (1998) suggested a reduction in SSW events in association with GW, Mahfouf et al. (1994) suggested an increase. The occurrence of SSW events is closely related to stratospheric circulation. Shindell et al. (1999) proposed that increased $\mathrm{CO}_{2}$ concentration enhances the subtropical jet (STJ), toward which tropospheric waves tend to be refracted, strengthening the polar vortex. Thompson et al. (2000) insisted that a strengthening trend was found in the stratosphere. However, Fyre et al. (1999) found no significant Arctic Oscillation trend until after year 2020 in atmosphere-ocean coupled GCM (AOGCM) experiments. The responses of the polar vortex and SSW to GW remain highly debated, with the results of GCMs not yet in agreement.

Here, we describe a GW projection and associated SSW events simulated using the Model for Interdisciplinary Research on Climate (MIROC) ${ }^{1}$ AOGCM. Among existing AOGCMs, only MIROC can achieve

Corresponding author: Graduate School of Science, Hokkaido University, Rigaku 8th Bilg., N10W8, Kita, Sapporo, Hokkaido 060-0810 Japan. E-mail: inaz@mail.sci.hokudai.ac.jp. @2007, the Meteorological Society of Japan.
Table 1 . The number of major $(+)$ and minor $(-)$ stratospheric sudden warmings (SSWs) counted every half-month in Oct-D ec and Jan-Apr for the 20th-century and 21st-century simulations (20CS and 21CS). The number is also counted for 44 winters in Sep 1958 to Aug 2002 based on the European Centre for Medium and Long-Range Weather Forecast Reanalysis Data (ERA-40). The bottom line shows the total number.

\begin{tabular}{|c|c|c|c|c|c|c|c|}
\hline & \multirow{2}{*}{ Period } & \multicolumn{2}{|c|}{$20 \mathrm{CS}$} & \multicolumn{2}{|c|}{$21 \mathrm{CS}$} & \multicolumn{2}{|c|}{ ERA40 } \\
\hline & & + & - & + & - & + & - \\
\hline Oct & $16-31$ & 0 & 0 & 0 & 1 & 0 & 0 \\
\hline Nov & $1-15$ & 0 & 0 & 1 & 0 & 0 & 0 \\
\hline Nov & $16-30$ & 0 & 0 & 0 & 0 & 2 & 3 \\
\hline Dec & $1-15$ & 0 & 0 & 1 & 1 & 3 & 2 \\
\hline Dec & $16-31$ & 0 & 0 & 2 & 2 & 1 & 6 \\
\hline Jan & $1-15$ & 0 & 2 & 5 & 4 & 4 & 4 \\
\hline Jan & $16-31$ & 1 & 1 & 2 & 2 & 2 & 10 \\
\hline Feb & $1-15$ & 3 & 4 & 9 & 12 & 2 & 15 \\
\hline Feb & $16-28(29)$ & 5 & 4 & 3 & 9 & 4 & 11 \\
\hline Mar & $1-15$ & 9 & 9 & 2 & 12 & 4 & 19 \\
\hline Mar & $16-31$ & 3 & 11 & 1 & 20 & 0 & 14 \\
\hline Apr & $1-15$ & 0 & 11 & 0 & 8 & 0 & 4 \\
\hline \multirow[t]{2}{*}{ Apr } & $16-30$ & 0 & 1 & 0 & 2 & 0 & 2 \\
\hline & Total & 22 & 43 & 26 & 75 & 22 & 90 \\
\hline
\end{tabular}

20th-century and 21st-century simulations (20CS and $21 \mathrm{CS})$ at $\mathrm{T} 106(\sim 100 \mathrm{~km})$ horizontal resolution and at 56 vertical levels in the atmosphere, a resolution sufficient to resolve mid-latitude transient waves in the troposphere and wave--mean flow interactions in the lower stratosphere. The two 100-yr integrations provide us ample SSW events to discuss seasonal variability. We expected this model to produce a plausible dataset for our purposes.

\section{Model experiments}

We used the MIROC 3.2 model, which contains five independent submodels for the atmosphere, ocean, land, rivers, and sea ice, and their coupling processes. The model has T106 ( 100-km grid size) resolution with 56 vertical levels for the atmosphere and $1 / 4^{\circ} \times 1 / 6^{\circ}$ and 48 vertical levels for ocean. The top level is set at 0.1 $\mathrm{hPa}$, and there are about 20 levels above $100 \mathrm{hPa}$. Atmospheric and oceanic dynamics are based on primitive equations. Major atmospheric and oceanic processes are parameterized, including radiative transfer, cumulus and oceanic convection, large-scale cloud condensation, turbulent mixing, and atmospheric gravity wave drags. The other submodels solve processes for snow, soil and canopy water, runoff, river flow, and sea ice. No flux adjustment is applied to the coupling. The technical report by the K-1 Model Developers (2004)

${ }^{1}$ Developed by Center for Climate System Research (CCSR), The University of Tokyo; National Institute for Environmental Studies (NIES) in Japan; and Frontier Research Center for Global Change (FRCGC), Japan Agency for Marine-Earth Science and Technology. 


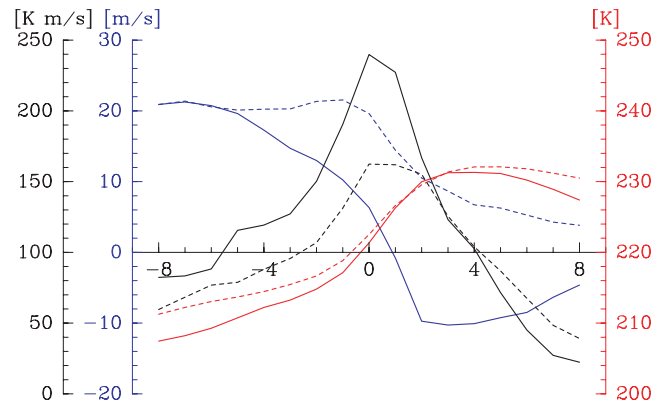

Fig. 1. Lead/lag time series of composite zonal-mean values of temperature at $80^{\circ} \mathrm{N}, 10 \mathrm{hPa}$ (unit $\mathrm{K}$; red lines; right scale); zonal wind at $65^{\circ} \mathrm{N}, 10 \mathrm{hPa}\left(\mathrm{m} \mathrm{s}^{-1}\right.$; blue lines; left blue scale); and total eddy heat flux (EHF) by meridional wind at $60^{\circ} \mathrm{N}, 100 \mathrm{hPa}$ ( $\mathrm{K} \mathrm{m} \mathrm{s}^{-1}$; black lines; leftmost scale). The composite analyses involved 22 major SSWs (solid lines) and 43 minor SSWs (broken lines) in 20CS. The horizontal axis shows the number of days led/lagged relative to the SSW onset date.

provides further details.

For the 20CS, we input historical data of atmospheric composition such as $\mathrm{CO}_{2}$ and ozone, from 1900 to 1999. The 21CS encompassed the years 2000 to 2099, with the estimated atmospheric composition given by scenario A1b, in which globalization and technology innovation greatly expand the global economy. The $\mathrm{CO}_{2}$ concentration level reaches $750 \mathrm{ppmv}$ in 2100 , but the stratospheric ozone does not change so much in the $21 \mathrm{st}$ century. We used daily data for these two 100-yr integrations.

\section{Methodology}

We here used the conventional definition of the SSW by the World Meteorological Organization. There are three conditions for the zonal-mean field at $10 \mathrm{hPa}$ : (A) a temperature at $85^{\circ} \mathrm{N}$ higher than that at $60^{\circ} \mathrm{N}$ for $>5$ days; (B) a change in zonal wind at $65^{\circ} \mathrm{N}$ to easterly; and (C) the restoration of zonal wind at $65^{\circ} \mathrm{N}$ from easterly to westerly at $>2 \mathrm{~m} \mathrm{~s}^{-1}$ within 30 days. A major SSW event satisfies all of these conditions. A minor SSW event satisfies condition A, but does not meet condition B. A "final" SSW event satisfies conditions A and B, but not condition C. Final SSW was omitted from our analysis. The onset date is defined as the first day that the conditions are satisfied.

In section 4 , the daily change in the eddy heat flux (EHF; $\propto$ the vertical component of EP flux) was discussed. Apart from this, for the logic in section 5, we used the suggestion that stratospheric extreme events are closely related to the lower stratospheric EHF when it is cumulated for the prior 40 days (Polvani and Waugh 2004), in order to discuss the relationship between SSW events and time-mean EHF at $100 \mathrm{hPa}$. Time-mean EHF can be divided into stationary- and transient-eddy contributions. Here we defined a stationary eddy as having fluctuation with a period of over 31 days (including climatology) and a transient eddy as having higher-frequency fluctuation. The EHF with all fluctuations is called the total EHF. The transient wave is here different from the storm-track wave discussed in section 5.3. This has a period of 2 to 8 days.

The refractive index as a function of zonal-mean zonal wind was used to diagnose the preferable direction of Rossby waves in section 5 . The index for waves with zonal wavenumber (ZWN) $s$ is given as Eq. (5.3.7) of Andrews et al. (1987). Waves tend to be refracted toward regions with a large index value and tend to be dissipated in regions with an imaginary value.
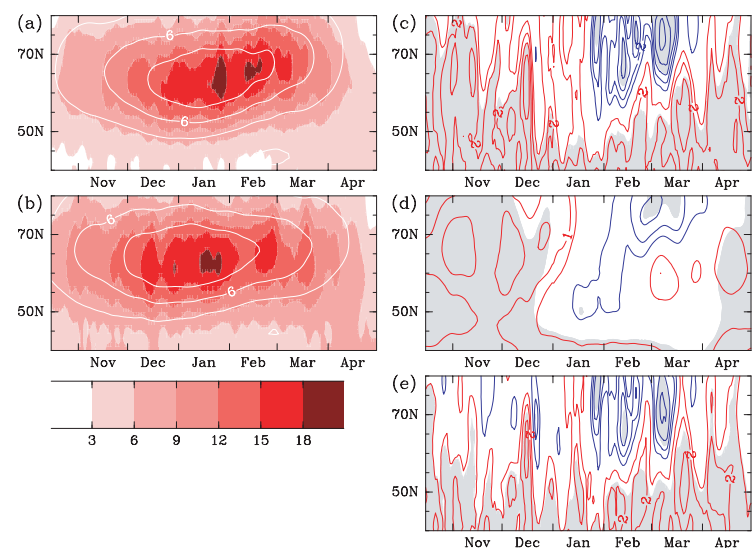

Fig. 2. (a and b) The seasonal progression of the zonal-mean EHF climatology at $100 \mathrm{hPa}$ for the (a) 20CS and (b) $21 \mathrm{CS}$. Contours show the stationary EHF at intervals of $3 \mathrm{~K} \mathrm{~m} \mathrm{~s}^{-1}$. As a reference, the shading shows total EHF to the right of (b). (c-e) Contours show the difference in (c) total, (d) stationary, and (e) transient EHF between the 20CS and 21CS. Contour interval is $1 \mathrm{~K} \mathrm{~m} \mathrm{~s}^{-1}$ for (c) and (e) and $0.5 \mathrm{~K} \mathrm{~m} \mathrm{~s}^{-1}$ for (d) with zero contour omitted and positive (negative) contours red(blue-) colored. Shadings denote the regions with > 95\% statistical signigicance level.

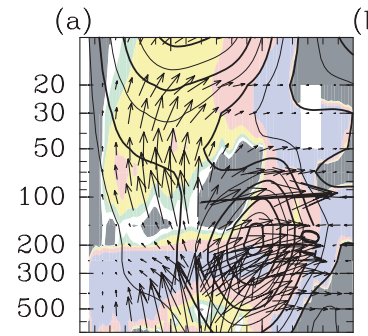

(b)
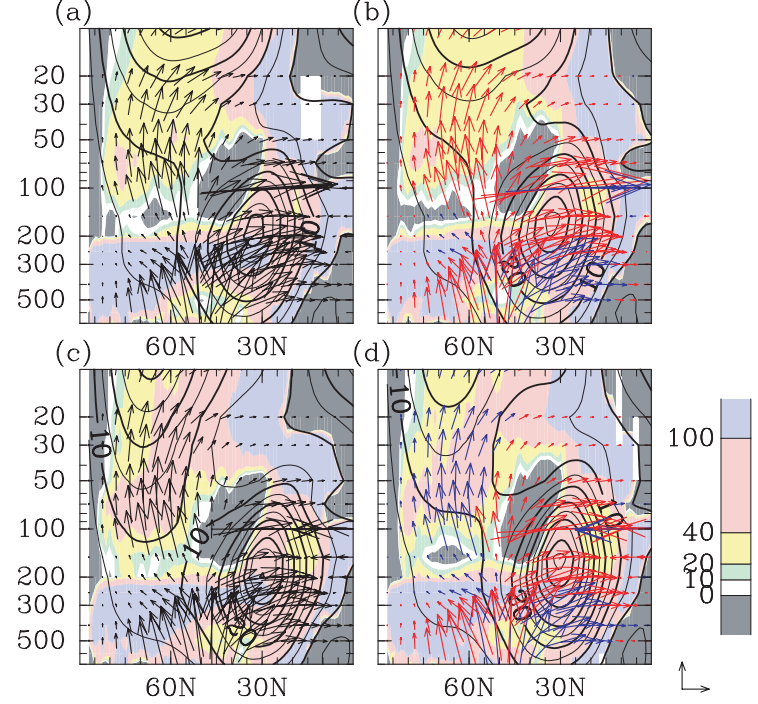

Fig. 3. Meridional cross-sections for the climatology of (a) Dec in the 20CS, (b) Dec in the 21CS, (c) Feb in the 20CS, and (d) Feb in the $21 \mathrm{CS}$. Contours: zonal-mean zonal wind at intervals of $5 \mathrm{~m} \mathrm{~s}^{-1}$. Vectors: Eliassen-Palm (EP) flux by total eddies with reference of $\left(5.0 \times 10^{7}, 5.0 \times 10^{5}\right) \mathrm{kg} \mathrm{s}^{-2}$ in the bottom right; the scale is increased tenfold above $100 \mathrm{hPa}$; in (b) and (d), red and blue shadings denote more and less vertical components of EP flux compared with those for the 20CS, respectively. Shading: the squared refractive index for $Z \mathrm{ZWN}-1$ waves, shown as the convention to the right.

\section{Simulated sudden warming}

First, we describe SSW events in the 20CS and compare these with observations. Table 1 shows the number of major and minor SSWs in every half-month periods. In the 20CS, there were 22 major and 43 minor SSW events in total. The SSW events, whether major or minor, mostly occurred in Feb and Mar, although they were observable throughout the winter (Table 1). The model underestimated the number of SSWs in Dec and Jan, with the frequency of modeled major SSW events 
half that of observed ones.

Using 22 major 20CS's SSW events (solid lines) or 43 minor ones (dashed ones), we calculated the lead/lag composite of zonal-mean values of polar temperature, wind in the polar night jet (PNJ), and total $\mathrm{EHF}$ at $60^{\circ} \mathrm{N}$ and $100 \mathrm{hPa}$ (Fig. 1). Eight days before the onset, similar to the midwinter climatology, the polar temperature was $<210 \mathrm{~K}$ and the PNJ wind was westerly at $>20$ $\mathrm{m} \mathrm{s}^{-1}$. Toward the onset, the polar temperature gradually increased and the PNJ weakened. At the onset, the composite total EHF in the lower stratosphere was maximized. The temperature then rapidly increased and, especially in the case of major SSW events, the wind direction reversed from westerly to easterly. The polar vortex was mostly shifted and was rarely split (not shown). These results demonstrate that the model can simulate SSW dynamics in which numerous Rossby waves propagating from the troposphere play an important role.

The underestimation of SSW events in Dec and Jan may be partly due to a negative temperature bias in the equatorial upper troposphere, which is related to slightly broader STJs in both meridional and vertical directions and a weaker PNJ (cf., Figs. 3 and $4 \mathrm{~b}, \mathrm{c}$ ). However, because it is difficult to obtain accurate distributions of zonal-mean wind by AOGCMs, and considering that atmospheric GCMs tend to miss SSW statistics (Charlton et al. 2007), our 20CS results can be regarded as a reasonable reference.

\section{Global warming projection}

\subsection{SSW statistics and dynamics}

The 21CS shows 26 major and 73 minor SSWs, a greater frequency than for the 20CS (Table 1). About $40 \%$ of the major SSWs are simulated to occur in Nov, Dec, and Jan in the 21CS, whereas only one major SSW occurs in these months in the 20CS. Adding the minor SSW events for these months gives five times more SSW events in the $21 \mathrm{CS}$ than in the 20CS. However, there is little difference between the simulation periods in Mar. The composite analysis indicates that the SSW dynamics remain similar in the $21 \mathrm{CS}$, with ZWN-1 waves propagating from the troposphere mainly contributing to the SSW events (not shown). We also checked that such GW response gradually starts emerging from the end of the 20th century (not shown).

\subsection{Wave-mean flow interaction in the stratosphere}

Following the idea by Polvani and Waugh $(2004)^{2}$, the increase in SSW events in Nov, Dec, and Jan can be explained using Figs. 2a-c. The shadings in Figs. $2 \mathrm{a}, \mathrm{b}$ represent the seasonal march of the climatological total $\mathrm{EHF}^{3}$ at $100 \mathrm{hPa}$. Here, daily climatology has been obtained through 5-day running mean of the 100-year average for each calendar day. In the 20CS (Fig. 2a), the $\mathrm{EHF}$ is $\sim 12 \mathrm{~K} \mathrm{~m} \mathrm{~s}^{-1}$ in Dec, and $\sim 18 \mathrm{~K} \mathrm{~m} \mathrm{~s}^{-1}$ in Feb, with a maximum latitude of approximately $60^{\circ}-70^{\circ} \mathrm{N}$ throughout the winter. In contrast, in the $21 \mathrm{CS}$ (Fig. $2 \mathrm{~b}$ ), the EHF is $\sim 15 \mathrm{~K} \mathrm{~m} \mathrm{~s}^{-1}$ in Dec and also in Feb, with its central latitude shifted slightly southward. The increase in the EHF in Nov, Dec, and early Jan corresponds to the increase in SSW events in Dec, Jan, and early Feb (Table 1). Figures $2 \mathrm{a}-\mathrm{c}$ show the decrease of the EHF in Feb, but the difference is not statistically significant. There is then little difference in the number of the SSW events in Mar, though the major SSW events decrease in Mar.

Figure 3 shows the EP flux vectors (tenfold scale above $100 \mathrm{hPa}$ ), the refractive index for $s=1$, and the zonal-mean zonal wind for the 20CS and 21CS. In Dec (Figs. 3a, b), zonal-mean wind is $>40 \mathrm{~m} \mathrm{~s}^{-1}$ in the STJ core and is $>25 \mathrm{~m} \mathrm{~s}^{-1}$ in stratospheric PNJ for both the $20 \mathrm{CS}$ and 21CS. Following zonal-mean wind, the wave prohibitation region extends around $45^{\circ} \mathrm{N}, 100 \mathrm{hPa}$ and sporadically exists at $65^{\circ} \mathrm{N}, 150 \mathrm{hPa}$ in both the $20 \mathrm{CS}$
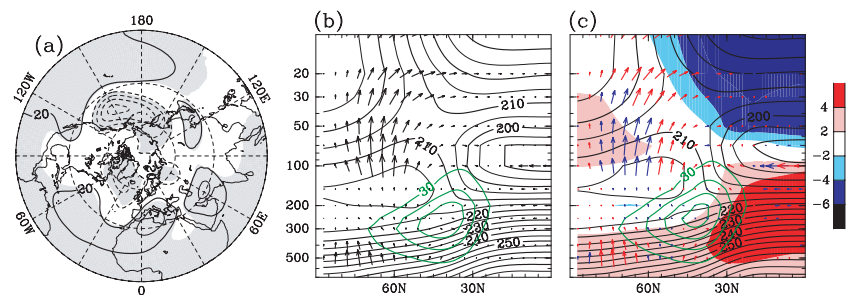

Fig. 4. (a) The difference in 300-hPa geopotential height in Dec between the $20 \mathrm{CS}$ and $21 \mathrm{CS}$, with global-mean surface uplift removed. The contour interval is $20 \mathrm{~m}$; the zero contour is omitted, and negative contours are shown as broken lines. The shading shows the regions with > 95\% statistical significance level. (b and c) Meridional cross-sections of Dec climatology for (b) the $20 \mathrm{CS}$ and (c) $21 \mathrm{CS}$. Black contour: zonal-mean temperature at intervals of $5 \mathrm{~K}$. Green contour: Zonal-mean storm-track wave kinetic energy. The contours show 30, 50, 70, and 90 $\mathrm{m}^{2} \mathrm{~s}^{-2}$. Vectors: the EP flux by transient waves with the ZWN-1 and ZWN-2 components. Colors are as in Fig. 3, but scales are fourfolds. Shading in (c): the difference in zonal-mean temperature between the $20 \mathrm{CS}$ and $21 \mathrm{CS}$, as the reference at the bottom right.

and $21 \mathrm{CS}$. The refractive index is quite similar around $65^{\circ} \mathrm{N}, 150 \mathrm{hPa}$, the most important region for the wave propagation to the stratosphere. The background state in $70-80^{\circ} \mathrm{N}, 150 \mathrm{hPa}$ is apparently different, but this region is not important for the wave propagation. The vertical component of EP flux is larger in the $21 \mathrm{CS}$ in both the stratosphere and troposphere. This is probably because there is increased wave activity in the troposphere (see section 5.3), though the background state does not seem to give a crucial effect to the wave propagation. The enhancement of the wave flux continues to early Jan and results in weakening of the PNJ in Feb.

For both 20CS and 21CS, seasonal changes can be found in the PNJ that is shifted northward and downward and in refractive index as well. In Feb (Figs. $3 \mathrm{c}, \mathrm{d}$ ), however, the 20CS and 21CS show different zonal-mean wind distributions in the polar stratosphere. The PNJ is still strong in the 20CS (Fig. 3c; $\sim 20 \mathrm{~m} \mathrm{~s}^{-1}$ at $60^{\circ} \mathrm{N}$ and $30 \mathrm{hPa}$ ), but weaker in the $21 \mathrm{CS}$ (Fig. $3 \mathrm{~d} ; \sim 15$ $\left.\mathrm{m} \mathrm{s}^{-1}\right)$. The weaker PNJ throughout the stratosphere holds a wave propagation prohibitation region around $65^{\circ} \mathrm{N}, 150 \mathrm{hPa}$ in the $21 \mathrm{CS}$. The weakening of the vertical curvature of zonal wind mainly contributes to the decreased refractive index [Eq. (5.3.7) in Andrews et al. (1987)]. The waves tend to be effectively blocked around this region. Moreover the upward elongation of the STJ and the meridional broadening of the lowerstratospheric westerlies induce the increase of refractive index around $50^{\circ} \mathrm{N}-60^{\circ} \mathrm{N}$ and $50-100 \mathrm{hPa}$. The increase leads to the enhanced upward propagation of Rossby waves, which is consistent with the southward shifted $\mathrm{EHF}$ in the lower stratosphere (Fig. 2).

\subsection{Wave sources in the troposphere}

In this section, we discuss the origin of wave responses for stationary and transient components. Contours in Figs. $2 \mathrm{a}$ and $\mathrm{b}$ show the stationary EHF at $100 \mathrm{hPa}$ in the $20 \mathrm{CS}$ and $21 \mathrm{CS}$, respectively. Figure $2 \mathrm{~d}$ indicates its difference. The flux difference in stationary EHF is positive in Dec and early Jan and negative in Feb. The ZWN-1 component of the flux is dominant (not shown).

${ }^{2}$ This idea may be supported by the notion that SSW tends to occur with a weak EHF anomaly, when the mean EHF is sufficiently large.

${ }^{3}\left[v^{*} \theta^{*}\right]=\left[v_{S}^{*} \theta_{S}^{*}\right]+\left[v_{T}^{*} \theta_{T}^{*}\right]+\left[v_{S}^{*} \theta_{T}^{*}\right]+\left[v_{T}^{*} \theta_{S}^{*}\right]$, where subscripts $\mathrm{S}$ and $\mathrm{T}$ represent stationary and transient components, respectively and the square bracket and the asterisk denote zonal mean and deviations from the zonal mean, respectively. The last two terms are dominant in the flux in Fig. 1, but are canceled out here by the time averaging. 
Figure $4 \mathrm{a}$ shows the response to GW at the $300-\mathrm{hPa}$ geopotential height in Dec. The Pacific-North Americanlike (PNA-like) pattern partially appears, presumably because Pacific tropical sea surface temperatures (SSTs) become more zonally uniform in the $21 \mathrm{CS}$. This response is actually related to the amplification of the stationary-eddy comoponent of ZWN-1 tropospheric waves $\left(103.6 \mathrm{~m}\right.$ to $110.1 \mathrm{~m}$ for $30^{\circ}-60^{\circ} \mathrm{N}$ average at 300 $\mathrm{hPa}$ ), which effectively propagate vertically. The stationary EHF (Fig. 2d) apparently shows the enhancement of the stationary wave propagation to the stratosphere in Dec. The hypothesis that the PNA response bridges tropical SSTs to SSWs may be analogous to the SSW response to the El Niño Southern Oscillation (Taguchi and Hartmann 2006). In Feb, a PNA-like response can be seen (ZWN-1 comoponent of stationary-eddy geopotential height increases $95.8 \mathrm{~m}$ to $100.1 \mathrm{~m}$ for $30^{\circ}-60^{\circ} \mathrm{N}$ average at $300 \mathrm{hPa}$ ), but the background state effectively blocks the amplified waves propagating from the troposphere (cf., Figs. 3c, d).

The transient EHF difference (Fig. 2e) is comparable to the stationary EHF difference (Fig. 2d), whereas the climatological contribution of transient EHF is of secondary importance (cf., Figs. 2a, b). The EHF difference is negative north of $60^{\circ} \mathrm{N}$, especially in Feb, but positive south of $60^{\circ} \mathrm{N}$. The transient EHF by ZWN-1 and ZWN-2 waves actually increases by about 15\% in Dec (Figs. 4 b, c).

The contours in Figs. $4 \mathrm{~b}$ and $\mathrm{c}$ show the storm-track activity (green lines) and zonal-mean temperature (black) in Dec for the 20CS and 21CS, respectively. The GW forcing increases the meridional temperature gradient because more latent heat is released in the tropical upper troposphere (Manabe and Wetherald 1975). The storm track prefers the gradient zone around the tropopause and is enhanced under $\mathrm{GW}^{4}$. Provided that mutual interaction among synoptic-scale eddies might enable storm eddies to transfer their energy to both transient and stationary large-scale eddies in high latitudes, the increase in transient ZWN-1 and ZWN-2 EHF might be explained. The flux by waves with ZWN $>3$ actually increases south of $60^{\circ} \mathrm{N}$, but such smallscale waves are evanescent above the tropopause. In Feb, although the storm track is also more active, there is less propagation of ZWN-1 and ZWN-2 waves because the basic state blocks wave propagation north of $60^{\circ} \mathrm{N}$ (cf., Figs. 3c, d).

However, it is difficult to assess why the storm-track activity increases in response to GW. A recent multimodel comparison of GW revealed increased stormtrack activity and a northward shift in the maximum latitude of this activity (Yin 2005). Such enhancement of storm activity is likely responsible for the change in the STJ. Our MIROC results suggest that the GW forcing strengthens the core of the STJ (Fig. 3). However, further investigations are needed to explore this process in detail.

\section{Conclusion and discussion}

We investigated SSW events under projected GW using the MIROC AOGCM. The 20CS represents SSW dynamics well, but misses SSW events in Nov, Dec, and Jan. Considering the difficulty in accurately simulating zonal-mean temperature using an AOGCM, we regard the $20 \mathrm{CS}$ as a reference. SSW events occur more frequently in Dec, Jan, and early Feb in the $21 \mathrm{CS}$, in close relation to EHFs by stationary and transient waves propagating from the troposphere in Nov, Dec, and early Jan. In Dec, a PNA-like response is associated with the amplification of ZWN-1 waves in tropospheric stationary eddies. More Rossby wave propagation in Dec and

${ }^{4}$ It is displaced northwards and may be related to the STJ enhancement. early Jan leads to the weaker PNJ in Feb under GW. The background state around $65^{\circ} \mathrm{N}, 150 \mathrm{hPa}$ effectively blocks the wave propagation in Feb, but the EHF difference is not statistically significant. Even so, the major SSW events in Mar decrease.

The results of a weaker polar vortex do not fit entirely with the findings of Shindell et al. (1999) mentioned in the introduction. This inconsistency may be related to model bias. The model that we used contains a bias of weaker PNJ and broader STJ, both of which probably influence wave propagation. Further model improvement and validation using observations are needed for more confident arguments.

\section{Acknowledgments}

We thank two anonymous reviewers for the improvement of this paper. This study was supported by the Frontier Research Project of The University of Tokyo, the Frontier Research Consortium on Climate and Environment Applications supported by the Itochu Co., Nippon Telegraph and Telephone Co., and Tokyo Marine \& Nichido Fire Insurance Co., Ltd., the Kyosei and Kakushin Projects of the CCSR, NIES, and FRCGC of the Ministry of Education, Culture, Sports, Science, and Technology in Japan. The model simulations were performed using the Earth Simulator, and the figures were produced using the GFD-Dennou Library.

\section{References}

Andrews, D. G., J. R. Holton, and C. B. Leovy, 1987: Middle Atmosphere Dynamics, Academic Press, $489 \mathrm{pp}$.

Charlton, A. J., L. M. Polvani, J. Perlwitz, F. Sassi, E. Manzini, K. Shibata, S. Pawson, J. E. Nielsen, and D. Rind, 2007: A new look at stratospheric sudden warmings. Part II. Evaluation of numerical model simulations, J. Climate, 20, 470488.

Fyre, J. C., G. J. Boer, and G. M. Flato, 1999: The Arctic and Antarctic oscillations and their projected changes under global warming, Geophys. Res. Lett., 26, 1601-1604.

K-1 Model Developers, 2004: K-1 coupled GCM (MIROC) description, H. Hasumi and S. Emori, Eds., K-1 Technical Report, 1, 34 pp.

Mahfouf, J. F., D. Cariolle, J.-F. Royer, J.-F. Geleyn, and B. Timbal, 1994: Response of the Meteo-France climate model to changes in $\mathrm{CO}_{2}$ and sea surface temperature, Clim. Dyn., 9, 345-362.

Manabe, S., and R. T. Wetherald, 1975: The effect of doubling the $\mathrm{CO}_{2}$ concentration on the climate of a general circulation model, J. Atmos. Sci., 32, 3-15.

Matsuno, T., 1971: A dynamical model of the stratospheric sudden warmings, J. Atmos. Sci., 28, 1479-1494.

Polvani, L. M., and D. W. Waugh, 2004: Upward wave activity flux as a precursor to extreme stratospheric events and subsequent anomalous surface weather regimes, $J$. Climate, 17, 3548-3554.

Rind, D., R. Suozzo, N. K. Balachandram, and M. J. Prather, 1990: Climate change and the middle atmosphere: Part I: The double $\mathrm{CO}_{2}$ climate, J. Atmos. Sci., 47, 475-494.

Rind, D., D. Shindell, P. Lonergan, and N. K. Balachandran, 1998: Climate change and the middle atmosphere. Part III: The doubled $\mathrm{CO}_{2}$ climate revisited, J. Climate, 11, 876-894.

Shindell, D. T., R. L. Miller, G. A. Schmidt, and L. Pandolfo, 1999: Simulation of recent northern winter climate trends by greenhouse-gas forcing, Nature, 399, 452-455.

Taguchi, M., and D. L. Hartmann, 2006: Increased occurrence of stratospheric sudden warmings during El Niño as simulated by WACCM, J. Climate, 19, 324-332.

Thompson, D. W. J., J. M. Wallace, and G. C. Hegerl, 2000: Annular modes in the extratropical circulation. Part II: Trends, J. Climate, 13, 1018-1036.

Yin, J. H., 2005: A consistent poleward shift of the storm tracks in simulations of 21 st century climate, Geophys. Res. Lett., 32, L18701.

Manuscript received 20 June 2007, accepted 9 October 2007 SOLA: http://www.jstage.jst.go.jp/browse/sola/ 